\title{
A photoferroelectric material is more than the sum of its parts
}

To the Editor - The defining property of ferroelectrics is a reversible spontaneous electric polarization whose magnitude and direction can be sensitively tuned by varying temperature, pressure, electric field, strain or chemical composition ${ }^{1}$. What makes ferroelectrics interesting is the coupling of the electric polarization to other properties of the material. For instance, ferroelectricferroelastic materials present a coupling between electric polarization and mechanical deformation, which can lead to a remarkable piezoelectric response with numerous applications for actuators and sensors. Another widely pursued materials class is that of ferroelectric-ferroelastic-magnetic materials, termed multiferroics, which present interesting coupling phenomena with high potential for electronic and spintronic applications. In this Correspondence we focus on another fascinating property of ferroelectric materials that is due to their interaction with light. Materials that show both photosensitive and ferroelectric properties define a field that was termed photoferroelectrics a long time $\mathrm{ago}^{2}$, but which has been largely overlooked and is now deserving of renewed attention.

The present revival of photoferroelectrics focuses on ferroelectric photovoltaic materials. Although photovoltaic effects in ferroelectrics have been known for 50 years ${ }^{2}$, they have remained an academic curiosity, mainly because of their reported low powerconversion efficiency. This view has recently changed following reports that the low conversion efficiencies can be overcome by large, above-bandgap photovoltages ${ }^{3}$, the possibility of tip-enhanced photovoltaic effects at the nanoscale ${ }^{4}$ or the fundamental role of domain walls, which present a much larger efficiency than the bulk ${ }^{3,5}$. All this indicates that ferroelectric photovoltaic materials potentially have a bright future in solar-energy generation. But how are we to separate fact from fiction, and hype from hope in discussing their potential?

One of the selling points for ferroelectric photovoltaics is the extremely large, abovebandgap open-circuit voltage, which points to a fundamentally different, polarizationrelated charge-separation mechanism compared with classical semiconductor solar cells. In addition, the presence of ferroelectric domain boundaries further increases the photovoltage significantly because of the electrical fields existing within the narrow domain walls $s^{3,5}$. Therefore, advances in this field could arise from investigating materials with engineered domain boundaries ${ }^{6}$, materials with an intrinsically complex landscape of the local electric polarization such as relaxor ferroelectrics, or complex oxides with an engineered bandgap.

Yet, how do photovoltaic ferroelectrics compare with other solar-cell technologies? To achieve a high power output a solar cell needs to show high photovoltage, high photocurrent, and of course high quantum efficiency. Unfortunately, in ferroelectric photovoltaics, quantum efficiencies remain at best on the order of $1 \%$ and bulk conductivities are also low. Similarly, the photovoltage arising from an individual domain wall, which is essentially an interface limited in width, is modest; high voltages will only originate cumulatively from a large series of domains. Significant efforts will be needed before ferroelectrics could reach similar performances to those of semiconductor solar cells. This may seem a daunting task, but we should not forget examples such as that of organic solar cells, which have increased their conversion efficiency from $1 \%$ to $10 \%$ in the past ten years.

But, in our view, such a focus on only photovoltaics is too restrictive, and we believe that more attention should be paid to other photoinduced effects in ferroelectrics. It is important to realize that photoinduced effects can, and usually will, be coupled to and with other functional properties. A good example of this is photostriction - the deformation induced by irradiation of light — which can be described in ferroelectrics as the combination of photovoltaic and piezoelectric effects. The photovoltaic effect creates an internal electric field, which in turn leads to significant deformation by the inverse piezoelectric effect. Light-induced size changes as recently reported ${ }^{7}$ for $\mathrm{BiFeO}_{3}$ single crystals can thus be understood from their ferroelectric properties and photovoltaic effects. Highly strained $\mathrm{BiFeO}_{3}$ thin films ${ }^{8}$ with enhanced piezoelectricity are likely to show even stronger photostrictive effects. $\mathrm{BiFeO}_{3}$ is also antiferromagnetic and it has been shown that its magnetic properties can be modified by both electric field and strain deformation, which presents the opportunity for also tuning the magnetic properties by photovoltage and photostriction.

This example illustrates the general principle and interest of having interactions between photoelectric effects and other (multi-)ferroic or correlated-electron effects such as charge order, metal-insulator phase transitions, electronic and magnetic phase separations and so on. The breadth of both possible photo-induced effects and correlated-electron physics in ferroelectrics is enormous, leaving us with a wide field of possible investigations into interesting physics and possible new applications, with the potential for remote (optical) control.

Finally, most of the recent work in the field focuses on the multiferroic perovskite $\mathrm{BiFeO}_{3}$, which is probably only the tip of the iceberg in terms of other interesting and useful materials. Investigations of photo-induced effects in multiferroics where magnetism causes ferroelectricity offer new degrees of freedom and coupling mechanisms, also on the ultrafast timescale. Generally speaking, the search for new interesting systems, be it in bulk form, thin films, clever nanostructures or domain-engineered materials, is crucial for a deeper understanding of photo-induced effects in ferroelectrics or more generally polar materials. Perhaps, beyond any hype on photovoltaic materials, it is rather on the broader family of photoferroelectrics that we should place most of our hope.

References

1. Wadhawan, V. K. Introduction to Ferroic Materials (Gordon and Breach, 2000).

2. Fridkin, V. M. Photoferroelectrics (Springer, 1979).

3. Yang, S. Y. et al. Nature Nanotech. 5, 143-147 (2010).

4. Alexe, M. \& Hesse, D. Nature Commun. 2, 256 (2011).

5. Seidel, J. et al. Phys. Rev. Lett. 107, 126805 (2011).

6. Salje, E. \& Zhang, H. L. Phase Transit. 82, 452-469 (2009)

7. Kundys, B., Viret, M., Colson, D. \& Kundys, D. O. Nature Mater. 9, 803-805 (2010).

8. Zeches, R. J. et al. Science 326, 977-980 (2009).

Acknowledgements

This Correspondence distills much of the discussion from a Workshop on Ferroelectric Photovoltaics - Fact, fiction, hype or hope? - held in January 2012 at the University of Warwick, UK.

J. Kreisel ${ }^{1 \star}$, M. Alexe ${ }^{2}$ and P. A. Thomas ${ }^{3}$

'Lab. Matériaux et Génie Physique, Minatec, CNRS, Grenoble Institute of Technology, 38016 Grenoble, France. ${ }^{2}$ Max Planck Institute of Microstructure Physics, 06120 Halle, Germany. 3Department of Physics, University of Warwick, Coventry CV4 7AL, UK.

*e-mail: Jens.Kreisel@grenoble-inp.fr 